\section{Experimentelle Untersuchungen zur Steigerung der Laktatdehydrogenase-Aktivität des Harnes}

Im Harn des Menschen und verschiedener Versuchstiere (Hund, Katze, Kaninchen, Meerschweinchen, Ratte) ist regelmässig eine Laktatdehydrogenase-(LDH-)Aktivität nachweisbar ${ }^{1}$. Zahlreiche Nierenerkrankungen bewirken eine Steigerung der LDH-Aktivität des Harnes; die Vermutung, dass vor allem maligne Prozesse im Bereich der Niere oder der ableitenden Harnwege zu einer erhöhten LDH-Ausscheidung führen ${ }^{2}$, hat sich nicht bestätigt ${ }^{1,3}$. $\mathrm{Da}$ die Rattenniere enzymhistochemisch ein weitgehend gleiches Verhalten der LDH zeigt wie die Niere des Menschen ${ }^{4}$ sollen im folgenden die Veränderungen der LDH-Aktivität des Rattenharnes nach Auslösung verschiedener Nierenschäden besprochen werden.

Matevial und Methoden. 1. Versuchstiere. Männliche Albinoratten (Sprague-Dawley) von $250 \mathrm{~g}$ Körpergewicht erhielten täglich $10,0 \mathrm{ml}$ Wasser mit der Schlundsonde verabreicht, um eine Ausscheidung möglichst gleichmässiger Harnmengen zu erzielen. Der Harn der Tiere wurde in Stoffwechselkäfigen aufgefangen und in 24-hPortionen untersucht. Die Bestimmung der normalen LDH-Aktivität im 24-h-Harn erfolgte bei 40 Ratten.

2. Nierenschädigung. (a) Vaskulärer Schock: Bei 10 Ratten wurde ein anaphylaktischer Schock ausgelöst (Antigen: Humanalbumin), bei weiteren 10 Tieren erfolgte die Auslösung eines anaphylaktoiden Schocks. Einzelheiten der Methodik wurden an anderer Stelle berichtet ${ }^{5}$.

(b) Toxische Nierenschädigung: 1,5 Ratten erhielten i.p. Injektionen von $250 \mathrm{mg}$ Natriumtetrathionat $/ \mathrm{kg}$ Körpergewicht (Lösungsmittel: physiologische Kochsalzlösung; Injektionsvolumen: 1,0 mi). Zehn Kontrolltiere erhielten 1,0 ml physiologischer Kochsalzlösung i.p.

(c) Hypokaliämische Nephrose (vgl, ${ }^{6}$ ): 10 Ratten wurden 14 Tage lang kaliumarm ernährt und erhielten zusätzlich noch einen Ionenaustauscher (Natrium-Polystyrensulfonat, K-Ionen-Entferner) mit der Schlundsonde verabreicht. (Einzelheiten der Methode sollen an anderer Stelle gemeinsam mit HoHENEGger publiziert werden.)

3. Bestimmung der LDH-Aktivität im Harn. Fünf $\mathrm{ml}$ der 24-h-Harnportion wurden $3 \mathrm{~h}$ gegen fliessendes Wasser dialysiert und anschliessend $15 \mathrm{~min}$ bei $3000 \mathrm{rpm}$ zentrifugiert. Die Bestimmung der LDH-Aktivität erfolgte mit der Testkombination der Fa, Boehringer, Mannheim. $0,1 \mathrm{ml}$ dialysierter Harn wurden mit $3,0 \mathrm{ml}$ $0,05 M$ Phosphatpuffer $\mathrm{pH} 7,5$ mit 0,0003 $M$ Pyruvat und mit $0,05 \mathrm{ml}$ einer $0,009 M$ wässrigen $\mathrm{NADH}-L o ̈ s u n g$ in einer Küvette zusammengemischt und die Extinktion bei $340 \mathrm{~nm}$ abgelesen. Nach $1 \mathrm{~min}$, nach $2 \mathrm{~min}$ und nach $3 \mathrm{~min}$ wurde erneut die Extinktion bestimmt. Die durchschnittliche Extinktionszunahme/min ergibt mit 5053 multipliziert die mU LDH-Aktivität in $1 \mathrm{ml}$ Harn. Sämtliche Ergebnisse wurden auf die 24-h-Harnmenge bezogen.

Ergebnisse. Die Ergebnisse sind in der Tabelle zusammengestellt. Die i.p. Injektion von $1,0 \mathrm{ml}$ physiologischer Kochsalzlösung bewirkt keine Anderung der LDH-Aktivität des Harnes (24-h-Wert).

Diskussion. Ebenso wie andere Enzymaktivitäten (vgl. ${ }^{5}$ und ${ }^{7}$ ) stammt auch die LDH-Aktivität des Harnes unter normalen Bedingungen aus den Tubuluszellen der Niere (Mauserung, Sekretion). Das Nierengewebe zeigt die höchste LDH-Aktivität aller Organe ${ }^{8,9}$. Beim vermehrten Zugrundegehen LDH-reicher Nierenepithelien, bei Störungen der Permeabilität dieser Zellen, bei Proteinurie (Ausscheidung von Serum-LDH) und bei Auftreten von Leukozyten oder Erythrozyten (LDH-reicher Blutzellen) im Harn kann eine Erhöhung der LDH-Aktivität des Harnes erfolgen (vgl. ${ }^{10}$ ).
Die LDH-Aktivität des Rattenharnes nach Auslösung verschiedener Nierenschäden

\begin{tabular}{|c|c|c|c|}
\hline $\begin{array}{l}\text { Versuchstiere } \\
\text { und Bedingungen }\end{array}$ & Zahl & $\begin{array}{l}\text { mU LDH- } \\
\text { Aktivität im } \\
\text { 24-h-Harn }\end{array}$ & Zunahme \\
\hline Normale Ratten & 40 & $117 \pm 23$ & - \\
\hline $\begin{array}{l}\text { Vaskulärer } \\
\text { Schock }\end{array}$ & 20 & $570 \pm 180$ & $\begin{array}{l}390 \% \\
(P<0,001 !)\end{array}$ \\
\hline $\begin{array}{l}\text { Natriumtetrathion } \\
\text { ohne Hämaturie }\end{array}$ & 10 & $731 \pm 170$ & $\begin{array}{l}520 \% \\
(P<0,001 !)\end{array}$ \\
\hline mit Hämaturie & 5 & $3410 \pm 1830$ & $2820 \%$ \\
\hline $\begin{array}{l}\text { Hypokaliämische } \\
\text { Nephrose }\end{array}$ & 10 & $543 \pm 210$ & $\begin{array}{l}360 \% \\
(P<0,005 !)\end{array}$ \\
\hline
\end{tabular}

Die hypoxische Nierenschädigung nach Auslösung eines vaskulären Schocks führt zu Permeabilitätsstörungen an den Tubuluszellen und zu vermehrtem Zugrundegehen der Nierenepithelien. Dies erklärt die Steigerung der LDH-Aktivität im Harn. Gleiche Uberlegungen gelten für die beobachtete Erhöhung der LDH-Aktivität des Harnes bei hypokaliämischer Nephrose. Nach toxischer Nierenschädigung durch Na-Tetrathionat (Modell nach 1I) setzt eine massive Proteinurie ein. Dies ist von einer starken Erhöhung der LDH-Aktivität des Harnes begleitet. Noch höhere Aktivitäten wurden nach Zustandekommen einer Hämaturie gemessen (Steigerung bis auf das Dreissigfache des Normalwertes).

Die angeführten Ergebnisse führen zu dem Schluss, dass bei der Ratte in gleicher Weise wie beim Menschen eine Erhöhung der LDH-Aktivität des Harnes bei vermehrtem Zugrundegehen oder bei Permeabilitätsstörungen der Tubuluszellen der Niere, bei Proteinurie und bei Auftreten LDH-reicher Blutzellen im Harn erfolgt. Die Art der zu derartigen Veränderungen führenden Noxen scheint ohne Bedeutung für das Zustandekommen der gesteigerten LDH-Ausscheidung im Harn.

Summary. In rat urine, determinations of lactate dehydrogenase $(\mathrm{LDH})$ activity were performed. Normal values ranged between 94 and $140 \mathrm{mU} / 24 \mathrm{~h}$ urine specimens. Experimentally induced renal damage (anaphylactic or anaphylactoid shock; administration of sodium. tetrathionate; potassium depletion) provoked a statistically significant increase in urinary LDH-activity. The 3 mechanisms by which such an increase might occur are briefly discussed.

W. RAAB

Universitäts-Institut für medizinische Chemie, A-1090 Wien (Österreich), 5. Mai 1967.

1 U. C. Dubach und R. Rediger, Urol. int. 17, 65 (1964).

${ }^{2}$ L. E. Dorfman, E. Amador und W. E. C. Wacker, J. am. Med. Assoc. 184, 1 (1963).

3 U. C. Dubach, Helv. med. Acta 33, 139 (1966).

U. C. Dubach, Klin. Wschr. 43, 753 (1965).

s. RaAB, Wien, klin. Wschr. 78, 364 (1966).

- B. Antoine, D. Patte und R. Barcelo, Actualités néphrol. (Hop. Necker, Flammarion, Paris 1962), p. 67.

T. RAAB, Experientia 22, 91 (1966).

8 F. Wroblewski und J. S. Ladue, Proc. Soc. exp. Biol. Med. 90, $210(1955)$.

Q U. C. DUbach, Klin. Wschr. 47, 157 (1963).

10 D. Amelung, H. D. Horn und E. Schröder, Klin. Wschr. 36, 963 (1958).

11 H. Bergmann und F. Truss, Medsche Welt, Stuttg. 7966, 1760. 